\title{
Avaliação cognitiva assistida para crianças com paralisia cerebral: o que informam as pesquisas?
}

\section{Erica Cindra de Lima Angela Donato Oliva}

Universidade da Força Aerea/ Universidade Estadual do Rio de Janeiro (UERJ)

E-mails: ericacindra@hotmail.com angeladonatoliva@gmail.com Instituto Brasileiro de Medicina de Reabilitação E-mail: rossanapugli@gmail.com Universidade Federal do Piauí (UFPI)

Universidade Estácio de Să E-mail: liviagviana@yahoo.com.br

Chistiane Delúsia de Oliveira Rocha E-mail: chistiane.delusia@gmail.com

Recebido em: 23 dezembro 2017. Aceito em: 27 março 2018. DOI: http://dx.doi.org/10.21674/2448-0479.43.380-396

\section{Resumo}

Este artigo de revisão oferece ao leitor uma síntese do conhecimento produzido, no período 20052015, sobre as melhores práticas em Avaliação Cognitiva Assistida, no atendimento de crianças diagnosticadas com Paralisia Cerebral - PC. Essa população infantil frequenta escolas, hospitais e Centros de Reabilitação. O conteúdo aqui proposto nos permite traçar o estado da arte sobre o tópico investigado. Como complemento, são explicitados os instrumentos, técnicas e procedimentos desta forma de avaliação. Quanto ao método, são empregados procedimentos de busca da Revisão Sistemática da Literatura (RSL), acessando-se bases de dados Psico Info, Cochrane Library, Portal Capes, BVS, Lilacs, Campbell Colaboration. A investigação se estendeu a artigos publicados em periódicos especializados e em relatórios de pesquisas recentes. Para cada base de dados foram detalhados procedimentos rotineiros em pesquisas de revisão, explicitando-se os critérios de inclusão e de exclusão. No total, foram encontrados, aproximadamente, 300 textos, no formato de artigos. Desse total, 10 foram incluídos e criticamente analisados, sustentando-se as hipóteses previamente formuladas. Os dados produzidos contribuem, sobremaneira, para a seleção criteriosa de instrumentos diagnósticos que compõem a Avaliação Cognitiva Assistida. É consolidado o papel das práticas baseadas em evidência, no atendimento de crianças com PC, com possibilidade de servirem de suporte para o pesquisador, na escolha das baterias de testes, dentre outros instrumentos diagnósticos que contemplem critérios de validade, fidedignidade e de padronização. Ações de Políticas Públicas poderão ser subsidiadas e direcionadas para a avaliação da aprendizagem das crianças afetadas.

Palavras-chave: Criança com Paralisia Cerebral. Avaliação Cognitiva Assistida. Evidência Cientifica. Prática Baseada em Evidência. Educação Especial. 


\section{Abstract \\ Cognitive assisted assessment for children with cerebral palsy: what research reports?}

This review article provides the reader with an overview of the knowledge produced in the period 2005-2015, on best practices in Assisted Cognitive Assessment in the care of children diagnosed with cerebral palsy - PC. This child population attends schools, hospitals and rehabilitation centers. The contents proposed here allows us to trace the state of the art on the topic investigated. As a complement, the tools, techniques and procedures of this form of assessment are explicit. As for the method, search procedures are employed of the Systematic Literature Review (RSL) by accessing databases Psycho Info, Cochrane Library, Capes Portal, BVS, Lilacs, Campbell Colaboration. The investigation was extended to articles published in professional journals and recent research reports. For each database were detailed routine procedures for review of research, is making explicit the inclusion and exclusion criteria. In total, it was found approximately 300 texts in the article format. Of these, 10 were included and critically analyzed, holding up the previously formulated hypotheses. The data produced contribute greatly to the careful selection of diagnostic instruments that make up the Cognitive Assessment Assisted. It consolidated the role of evidence-based practices in the care of children with $\mathrm{CP}$, with the possibility of serving as a basis for the researcher, the choice of test batteries, among other diagnostic instruments dealing with criteria of validity, reliability and standardization. Public Policy actions may be subsidized and directed to assessing the learning of the affected children.

Keywords: Children with Cerebral Palsy. Assisted Cognitive Assessment. Scientific Evidence. Evidence-Based Practice. Special Education.

\section{Introdução}

Durante a última década, a literatura científica registrou um volume reduzido de pesquisas publicadas sobre o uso da Avaliação Cognitiva Assistida no processo diagnóstico de crianças com Paralisia Cerebral (PC). Quando se investiga avaliação da aprendizagem para esta clientela a maioria dos artigos apresentam as avaliações normativas padronizadas e poucos pesquisadores utilizam-se da avaliação assistida em seus estudos, por isso a literatura que foque na avaliação cognitiva assistida ainda é escassa. A Avaliação Cognitiva Assistida é caracterizada por ser um conjunto de estratégias instrucionais utilizadas durante o processo de avaliação, a fim de garantir o fornecimento de ajuda, melhorando as condições da situação de avaliação, para que a criança possa revelar seu desempenho potencial e, em situações de resolução de problemas, possa atingir um grau crescente de autonomia (LINHARES, 1995). Dessa forma, a Avaliação Cognitiva Assistida é dinâmica, interativa e inclui a intervenção de ensino, promovida por um examinador mais experiente durante todo o 
processo de avaliação (LINHARES et al., 1998). Além disso, neste tipo de avaliação, afere-se o quanto as crianças podem evoluir em suas estratégias de resolução de problemas frente à "otimização" da situação de avaliação pela examinadora, através de suporte de ajuda instrucional, temporário e ajustável às suas necessidades.

Obviamente, a comunidade científica reconhece a relevância de se produzir conhecimentos inovadores que atendam as demandas da população infantil, notadamente, daquela com necessidades educativas especiais. Nessa perspectiva, os estudiosos e pesquisadores da Educação/Educação Especial clamam pela urgência na adoção de estratégias pedagógicas e psicológicas atualizadas para fins de avaliação "assistida", como também para a avaliação "dinâmica" (HAYWOOD; TZURIEL, 2002).

Nessa perspectiva, este artigo de revisão tem como objetivo apresentar os resultados de uma pesquisa direcionada para a verificação da evidência científica dos resultados gerados por procedimentos avaliativos, no âmbito da Avaliação Cognitiva Assistida. Essas pesquisas revisadas foram conduzidas por profissionais que atuam com crianças diagnosticadas com Paralisia Cerebral PC. Os resultados da revisão revelaram também os indicadores da eficácia diagnóstica para esse contingente infantil com necessidades especiais.

A Avaliação Diagnóstica ocupa lugar de destaque e oferece subsídios ao planejamento das estratégias pedagógicas no contexto geral do processo educativo de crianças, inclusive daquelas com diagnóstico de Paralisia Cerebral - PC. Os benefícios da avaliação diagnóstica cumprem a tarefa de melhoria do atendimento das necessidades e das características comportamentais e atitudinais dessa população especial (TONINI, 2012).

Os dados gerados por essa modalidade de avaliação permitem ao profissional da educação tomar decisões pertinentes à instalação de repertórios de comportamentos adaptativos que realmente promovam a inserção/inclusão da criança com PC, no ambiente físico e social, juntamente com seus pares e os membros da própria família. Com esse entendimento, a avaliação não se constitui em apenas um processo desvinculado da intervenção, mas sim que a antecede (MANZINI, 1999). As baterias de testes psicopedagógicos, destinadas à Avaliação Diagnóstica de crianças com PC, geram dados extremamente úteis para o educador no processo decisório da escolha das modalidades de ensino da linguagem, programas de desenvolvimento de habilidades comunicativas e também de aquisição de competências sociais. Além disso, os resultados produzidos por essas baterias de testes são de significativa relevância, tanto na gestão do processo de aprendizagem escolar, como também no manejo do comportamento em ambientes diversos, frequentados pela criança. Infelizmente, nem sempre essas ferramentas avaliativas encontram-se devidamente adaptadas quando aplicadas na população com necessidades educativas especiais (ROSS; CRESS, 2006; DELGRACIA, 2007). Vale assinalar que o processo de Avaliação Cognitiva Assistida consiste em uma modalidade de avaliação fundamentada na abordagem sócio-construtivista do desenvolvimento cognitivo (VYGOTSKY, 1988), particularmente envolvendo os conceitos de Zona de Desenvolvimento Proximal ${ }^{1}$ e de aprendizagem

\footnotetext{
1 A ZDP consiste na "distância que medeia entre o nível de desenvolvimento atual da criança, determinado pela sua capacidade de resolver problemas individualmente e o nível de desenvolvimento potencial, determinado através da resolução de problemas sob a orientação de adultos ou em colaboração com os pares mais capazes" (Vygotsky. 1984). 
mediada ${ }^{2}$. A avaliação assistida é dinâmica, interativa e inclui três etapas distintas: a fase inicial sem ajuda, assistência e manutenção (LINHARES, 1995). Na fase inicial sem ajuda, cria-se uma situação de avaliação para ver o que a criança sabe; na fase de assistência, o mediador ajuda a criança, em um processo parecido com o de ensino, até que ela se torne capaz de resolver o problema dado; na fase de manutenção a criança passa por uma nova avaliação em que deve resolver o problema sozinha. "Essa avaliação mostra a sensibilidade da criança à ajuda, como ela responde e também indica de que tipo de apoio ela precisa" (ENUMO, 2006).

$\mathrm{Na}$ fase de assistência, inclui-se o suporte de ajuda com pistas graduadas e padronizadas, derivadas da análise da tarefa em um determinado domínio cognitivo, possibilitando verificar-se a sensibilidade do examinando à instrução, através do número de pistas que necessita para completar a tarefa (RUTLAND; CAMPBELL, 1996). Embora ocorram diferenças entre as avaliações psicométrica e assistida, há uma tendência em utilizá-las de forma combinada, reunindo-se as informações específicas advindas de cada técnica de modo complementar (DIAS; PAULA; ENUMO; FERRÃO, 2011; DIAS; CUNHA; ENUMO, 2010; TURRINI; ENUMO; FERRÃO; MONTEIRO, 2010; DIAS; PAULA; ENUMO, 2010; DIAS; PAULA; ENUMO, 2009; PAULA; ENUMO, 2007; GERA; LINHARES, 2004). A avaliação assistida tem sido recomendada para crianças que apresentam dificuldades de aprendizagem, deficiências cognitivas ou desvantagens culturais (DIAS; PAULA; ENUMO; FERRÃO, 2011; DIAS; CUNHA; ENUMO, 2010; TURRINI; ENUMO; FERRÃO; MONTEIRO, 2010; DIAS; PAULA; ENUMO, 2010).

No que se refere ao uso da avaliação diagnóstica de crianças com PC, cabe ressaltar que a aplicação de rótulos diagnósticos não se constitui tarefa fácil entre pesquisadores, especialistas e educadores dedicados aos assuntos sobre Paralisia Cerebral. Há relatos que, nessas crianças, a alteração mais evidente é o déficit do comprometimento motor, ocasionando, assim, modificações diversas e consequentes perturbações na biomecânica da marcha e da postura corporal. Além disso, a criança poderá apresentar alterações nos processos cognitivos, incluindo-se o pensamento, a memória, a percepção, a atenção, dentre outros. Alterações poderão ocorrer também sob a forma de deficiências sensoriais, como por exemplo, as visuais, as auditivas, as olfativas, dentre outras. Reunindo-se restrições cognitivas, sensoriais e motoras, essas contribuem sobremaneira para déficits no desempenho funcional das crianças diagnosticadas com Paralisia Cerebral (OLIVEIRA, 2000; KNOX; EVANS, 2002; SCHWARTZMAN, 2004; KAVCIC; VODUSEK, 2005). Cabe ressaltar, porém, que a criança com $\mathrm{PC}$ exibe diferentes características comportamentais, no entanto, essa concepção não implicaria, necessariamente, na presença de déficits cognitivos ou potencial limitado para

\footnotetext{
${ }^{2}$ A Experiência da Aprendizagem Mediada (EAM) é a uma interação na qual o mediador se situa entre o organismo do indivíduo mediado e os estímulos de forma a selecioná-los, ampliá-los ou interpretá-los utilizando estratégias interativas para produzir significações além das necessidades imediatas da situação. O aprendiz não se beneficia somente da exposição direta a um estímulo em particular, mas cria, a partir dela, orientações, atitudes e técnicas que o modifica. A EAM é o que determina a flexibilidade que afeta o indivíduo de maneira significativa produzindo a plasticidade da inteligência.

Para Feuerstein, há um mediador, necessariamente um ser humano, que seleciona, filtra, organiza, nomeia e dá significado aos objetos. O mediador transmite sua visão do mundo e, antes que o mediado estabeleça sua própria visão, ele equilibra o seu conhecimento com o saber do mediador.
} 
aprender e se adaptar a diferentes ambientes. Pelo contrário, a criança com PC, quando recebe atendimento apropriado, desde tenra idade, pode demonstrar desempenho acadêmico de acordo com suas habilidades cognitivas (LAYTON; LOCK, 2001).

Ocorre que os responsáveis pelo processo diagnóstico e pelo atendimento dessa clientela especial carecem de preparo técnico-pedagógico e, além disso, observa-se escassez ou até mesmo falta de instrumentos adequados no mercado, para fins de avaliações diagnósticas das suas habilidades cognitivas. Essa é uma evidência empírica, compartilhada entre os profissionais da área. Ainda por esses motivos, as crianças com PC costumam apresentar baixo desempenho quando submetidas ao processo de avaliação psicopedagógica. Esse baixo rendimento no desempenho, acontece possivelmente pelas dificuldades dos profissionais em trabalhar com baterias de testes ajustadas aos preceitos psicométricos (DIAS; PAULA; ENUMO; FERRÃO, 2011; DIAS; CUNHA; ENUMO, 2010; TURRINI; ENUMO; FERRÃO; MONTEIRO, 2010; DIAS; PAULA; ENUMO, 2010; DIAS; PAULA; ENUMO, 2009; PAULA; ENUMO, 2007; GERA; LINHARES, 2004). Mais ainda, são questionáveis os tipos de ambientes nos quais essas crianças são avaliadas, considerando-se seus déficits motores e sensoriais. A avaliação é também prejudicada tendo em vista que as crianças com PC não oralizadas estão impedidas de se comunicar por meio da fala articulada (PAURA, 2009).

Para realizar uma boa avaliação é preciso levar em conta as características do contexto de aplicação, o que se pretende avaliar, a adequação dos instrumentos e das técnicas a serem utilizadas, assim como, atentar para as condições técnicas, metodológicas e operacionais do instrumento ou técnica a ser utilizada (NORONHA, 2013). Portanto, é necessária a escolha adequada, assim como, o modo de utilização dos procedimentos diagnósticos endereçados à população afetada. Obviamente, esses recursos deverão se adequar às demandas dessa clientela com deficiência, incluindo-se, por exemplo, a avaliação "assistida" ou avaliação "dinâmica" (HAYWOOD; TZURIEL, 2002; TZURIEL, 2001). Nessa perspectiva, os resultados das pesquisas sobre a avaliação assistida vêm delineando um campo promissor. Trata-se de estratégia inovadora, o que a distingue de uma avaliação tradicional, o que favorece 0 atendimento da população que apresenta dificuldades no processo de aprendizagem ou aquela em situação de desvantagem social (OMOTE, 2004; GERA; LINHARES, 2004; PAULA; ENUMO, 2007; ENUMO, 2009; TURRINI; ENUMO; FERRÃO; MONTEIRO, 2010; DIAS; PAULA; ENUMO, 2010; QUEIROZ, 2011; BRIDGES CATTS, 2011; KANTOR WAGNER; TORGESEN; RASHOTTE, 2011; DIAS; PAULA; ENUMO; FERRÃO, 2011; STAINBACK; OLSWANG; FEUERSTEIN; PINDER; DOWDEN, 2013; QUEIROZ; ENUMO; PRIMI, 2013).

Embora não seja consensual, as concepções sobre Avaliação Assistida apresentam pontos convergentes. No presente artigo, esse procedimento diagnóstico é conceituado como instrumento sensível para avaliar de forma mais ampla e prescritiva, crianças com déficits comunicativos, como aquelas com PC. (GERA; LINHARES, 2004; PAULA; ENUMO, 2007; DIAS, PAULA; ENUMO, 2009; DIAS; CUNHA; ENUMO, 2010; TURRINI; ENUMO; FERRÃO; MONTEIRO, 2010; DIAS; PAULA; ENUMO, 2010; OLIVEIRA; ENUMO; QUEIROZ, 2011; BRIDGES CATTS, 2011; KANTOR WAGNER, TORGESEN; RASHOTTE, 2011; DIAS; PAULA; ENUMO; FERRÃO, 2011; OLSWANG; FEUERSTEIN; PINDER; DOWDEN, 2013; QUEIROZ; ENUMO; PRIMI, 2013). 
Tendo-se como suporte os trabalhos de pesquisa citados nessa breve introdução do artigo, foram formuladas as seguintes hipóteses:

- Os profissionais que atuam com crianças com Paralisia Cerebral não utilizam a Avaliação Cognitiva Assistida para fins de diagnóstico.

- A literatura científica registra um volume reduzido de pesquisas publicadas sobre a Avaliação Cognitiva Assistida, no processo diagnóstico de crianças com Paralisia Cerebral (PC).

- Os resultados da Avaliação Cognitiva Assistida permitem ao profissional da educação tomar decisões pertinentes à instalação de repertórios de comportamentos adaptativos nas crianças com PC.

- Os benefícios da avaliação diagnóstica cumprem a tarefa de melhoria do atendimento das crianças com Paralisia Cerebral.

\section{Paralisia Cerebral}

A Paralisia Cerebral (PC), enquanto entidade nosológica que afeta esse contingente de crianças, é registrada nos sistemas oficiais de classificação e uso de rótulos (DSM V, CID 10), como um conjunto não progressivo de desordens do desenvolvimento, movimento e da postura, descritos como síndromes do desenvolvimento motor secundário a lesões ou anomalias decorrentes do cérebro, na fase inicial do seu desenvolvimento. É, de fato, um sintoma complexo com vários tipos e graus de envolvimento motor, originados em distúrbios não progressivos que ocorrem no cérebro em desenvolvimento. As desordens motoras da PC são geralmente acompanhadas por alterações na sensação, percepção, cognição, comunicação, comportamento e também por crises convulsivas (ROSEBAUM; PANETH; LEVITON; GOLDSTEIN; BAX, 2007)

Estudos epidemiológicos sugerem que a incidência da PC nos países desenvolvidos gira em torno de 2 a 3 por 1000 nascidos vivos. Nos países subdesenvolvidos, a incidência é maior, com um índice de 7 por 1.000 nascidos vivos. No Brasil, a estimativa é de cerca de 30.000 a 40.000 novos casos por ano (ZANINI; CEMIN; PERALLES, 2009; FONSECA, 2011; MORENO-DE-LUCA, 2012).

São múltiplos os fatores contribuintes para o desenvolvimento da PC e suas manifestações de sintomas podem ocorrer no período pré-natal, perinatal ou pós-natal, mas há indícios de que $70 \%$ a $80 \%$ sejam de origem pré-natal. Essas causas podem ser congênitas, genéticas, inflamatórias, infecciosas, anóxicas, traumáticas e metabólicas. $O$ baixo peso ao nascer e a prematuridade aumentam, significativamente, a possibilidade de uma criança desenvolver Paralisia Cerebral (CANS, 2000; MACGILLIVRAY; CAMPBELL, 1995; PHAROAH; COOKE; ROSENBLOOM, 1987; STANLEY; WATSONI, 1992; HÁGBERG et al., 1984, MURPHY; HOPE; JOHNSON, 1997).

Resultados de pesquisas recentes sustentam que a falta de acesso a serviços adequados de saúde, tanto no período pré-natal, quanto à assistência inadequada à mãe e ao bebê, nos períodos peri e pós-natal, aumentam a incidência da Paralisia Cerebral (SERDAROGLU; CANSU; ÕZKAN; TEZCAN, 2006; JOHNSON, 2002; WESTBOM; HAGGLUND; NORDMARK, 2007; BLUME; LOCH; LI, 
2007; VOHR; MSALL; WILSON; WRIGTH; MCDONALD; POOPLE, 2005; ROBERTSON; WATT; YASUI, 2007). O maior índice de Paralisia Cerebral encontra-se entre as crianças com extremo baixo peso e prematuridade, ocorrendo em torno de 19\% dos casos (SILVEIRA; PROCIANOY, 2005).

No contexto escolar, quando se reporta à criança com Paralisia Cerebral (PC), a sua especificidade de grau e de manifestações que frequentemente estão associados, se pressupõe necessidade de medidas educativas diversificadas. $O$ ingresso de um aluno com PC no ensino regular significa, frequentemente, o confronto com barreiras físicas, sensoriais e organizacionais, que dificultam ou impedem o acesso às instalações, à leitura/escrita, às tecnologias digitais ou a uma estrutura curricular adequada (MARTINS; LEITÃO; LETÍCIA, 2012; DÍAZ; BORDAS; GALVÃO; MIRANDA, 2009). Assim, as práticas pedagógicas, apesar de se aproximarem dos modelos estabelecidos pelo enquadramento legislativo, encontram ainda barreiras e pré-conceitos em estratégias de diferenciação pré-determinadas e, não raro, circunscritas à reprodução de programas previamente definidos (LEITÃO, 2007).

Supostamente, o que agrava a condição de excepcionalidade do aluno com PC e dos demais com necessidades educacionais especiais é talvez o modo como esses são concebidos pela própria sociedade, notadamente acerca de seu desempenho social e escolar. Um dos principais préconceitos em relação aos alunos com PC aponta para o fato do repertório de comportamento dessas crianças ser, erroneamente, associado a perturbações intelectuais e enquadrados em medidas educativas restritivas. Esta situação decorre, frequentemente, do fato de não Ihes ter sido ofertada uma avaliação que traduza, com fidelidade, as suas especificidades. Evidencia-se assim o processo dialético de exclusão/inclusão social perversa do qual são vítimas. Para Sawaia (2001), trata-se de um processo complexo fundado em três dimensões: a dimensão objetiva, mediante a desigualdade social, a dimensão ética através das injustiças e a dimensão subjetiva através do sofrimento psíquico.

O atual cenário evidencia um grau de desempenho escolar comprometido por total ausência de ações psicopedagógicas eficazes, como por exemplo, definição de critérios avaliativos de desempenho do aluno (FERNANDES, 2014; BORGES, 2011; HUANGA; DIAMOND, 2009; PEETERS; MOORR; VERHOEVEN, 2011). Nesse sentido, o presente trabalho visa identificar, por meio de uma revisão sistemática da literatura, como os atuais estudos relatam as melhores práticas em Avaliação Cognitiva Assistida no atendimento de crianças diagnosticadas com paralisia cerebral.

\section{Material e Métodos}

Trata-se de pesquisa exploratória, qualitativa e descritiva, com utilização de procedimentos metodológicos da Revisão Sistemática da Literatura (RSL) operacionalizada através de procedimentos de busca eletrônica, conduzidos em nível nacional e internacional. Para tanto, foram acessadas as bases de dados Psico Info, Cochrane Library, Portal Capes, BVS, Lilacs, Campbell Colaboration. Com o objetivo de padronizar a linguagem de busca, dentre as diversas bases de dados eletrônicas, foram utilizados os descritores de saúde DeCS e Mesh. Nessa estratégia de 
busca, os descritores e termos livres foram escritos "Avaliação cognitiva assistida" and"criança com paralisia cerebral", "dynamic assessment" and cerebral palsy and Assessment cognitive assistive and cerebral palsy. Esses descritores foram utilizados isoladamente ou combinados entre si, com o auxílio dos operadores booleanos $A N D$ e $O R$, de acordo com as especificidades de cada base de dados pesquisada, no período de maio a junho de 2015.

A pergunta formulada que norteia a presente pesquisa é a seguinte: Quais as técnicas e os procedimentos atualmente utilizados para fins de Avaliação Cognitiva Assistida de crianças com Paralisia Cerebral? A pergunta complementar é: Qual o grau de eficácia desses modos de avaliação quando aplicados neste contingente infantil?

O trabalho de pesquisa seguiu as mesmas fases propostas em pesquisas de revisão, quais sejam: a) explicitação do problema a ser investigado; b) formulação da pergunta norteadora da pesquisa; c) rastreamento nas bases de dados disponíveis; d) coleta e análise crítica dos resultados das pesquisas incluídas; e) discussão dos resultados encontrados e f) apresentação de quadro sintético dos resultados da Revisão Sistemática da Literatura.

\section{CRITÉRIOS DE INCLUSÃO E DE EXCLUSÃO: PROCEDIMENTOS}

As RSL recuperadas foram analisadas em relação aos critérios de inclusão. Os títulos e resumos dos artigos foram analisados, sendo considerados passíveis de inclusão, na Revisão Sistemática e o artigo que teve como resposta afirmativa em todas as 3 (três) questões:

- O conteúdo do artigo aborda as técnicas e os procedimentos da Avaliação Cognitiva Assistida?

- Os resultados conclusivos das pesquisas evidenciavam o grau de eficácia dos procedimentos e técnicas utilizados na Avaliação Cognitiva Assistida?

- Os resultados das pesquisas conferem com os objetivos pretendidos pelos pesquisadores?

Foram excluídos da pesquisa os estudos que obtiveram resposta negativa, em qualquer uma das três questões formuladas, não correspondendo, portanto, ao conteúdo de interesse da pergunta inicial.

De posse das informações coletadas e minuciosamente extraídas dos artigos analisados, foi possível consolidar dados para efetuar a apresentação de maneira consistente e não enviesada, conforme apresentado a seguir.

\section{COLETA E ANÁLISE DOS DADOS}

Inicialmente, cada RSL foi submetida à primeira avaliação dos títulos, seguida de leitura integral do resumo, eliminados quando não se enquadravam nos critérios de busca previamente determinados. Os dados foram extraídos da Revisão Sistemática da Literatura, sob a perspectiva qualitativa e expostos no formato de relatório descritivo, conforme tabela. Para o registro dos dados 
da RSL, foram elaborados quadros contendo as seguintes informações: autores, ano de publicação, procedimentos de avaliação assistida, número de participantes, procedimento de avaliação psicométrica e resultados.

\section{Resultados e Discussão}

A tabela abaixo apresenta a análise dos autores e o procedimento de avaliação assistida realizado.

$\begin{array}{ll}\text { Autores } & \text { Procedimento de avaliação Assistida/ Procedimento de } \\ \text { Avaliação Psicométrica/ Resultados }\end{array}$

1. LSWANG, FEUERSTEIN, PINDER, DOWDEN, 2013
Bayley Scales of Infant and Toddler Development, Third Edition (BSID-3 ; Bayley, 1993) Visual Reception Subtest from the Mullen Scales of Early Learning (MSEL, Mullen, 1995) / Houve associações significativas entre o desempenho nas provas assistidas e psicométricas, confirmando-se a proposição de complementaridade dessas metodologias avaliativas

2. QUEIROZ, ENUMO, Medidas de consciência fonológica/ Matrizes Progressivas PRIMI, 2013

Coloridas de Raven- Raven-MPC (ANGELINI et al., 1999) /Escala de Maturidade Mental Columbia - EMC (Burgeimester, Blum \& Lorge, 1999)/ Não houve associações significativas entre o desempenho nas provas assistidas e psicométricas, confirmando-se a proposição de complementaridade dessas metodologias avaliativas.

3.BRIDGES, CATTS, Static Deletion Task (SDT) Dynamic Screenin GO Phonological 2011

Awareness (DSPA) Initial Sound Fluency (ISF) WRMT-R/NU Word Identification WRMT-R/NU Word Attack/ Os resultados fornecem suporte preliminar para a utilidade de uma medida de rastreio dinâmico da consciência fonológica para os alunos do jardim de infância.

4. OLIVEIRA, ENUMO, Children's Analogical Thinking Modifiability- CATM (Tzuriel\& QUEIROZ, 2011 Klein, 1990) / Instrumento de Avaliação do Repertório Básico para Alfabetização - IAR (Leite, 1984)/ Child Behavior Checklist (CBCL 11/2-5 anos) (Achenbach \& Rescorla, 2004/ (CBCL 11/2-5 anos) (Achenbach \& Rescorla, 2004) Teste de Vocabulário por Imagens Peabody - TVIP (Capovilla \& Capovilla, 1997) Escala Columbia de Maturidade Intelectual (Burgmeister, Blum\&Lorge, 2001

5. KANTOR, WAGNER, Não informado /Não houve associações significativas entre o TORGESEN,RASHOTTE, desempenho nas provas assistidas e psicométricas, 2011 confirmando-se a proposição de complementaridade dessas 
metodologias avaliativas.

6. DIAS, PAULA ENUMO, Children's Analogical Thinking Modifiability Test - CATM (Tzuriel FERRÃO, 2011 e Klein, 1990)/ Escala de Comunicação Pré-Verbal, Lista de Avaliação da Linguagem Expressiva - LAVE (Capovilla e Capovilla, 1997) Matrizes Progressivas Coloridas de Raven Escala Especial (Angelini, Alves, Custódio, Duarte e Duarte, 1999)

7. TURRINI, ENUMO, Prova cognitiva assistida Children's Analogical Thinking FERRÃO MONTEIRO, Modifiability - CATM (TZURIEL; KLEIN, 1990) / Os resultados 2011 corroboram a literatura relacionando fatores afetivomotivacionais e desempenho cognitivo a prematuridade e o baixo peso com riscos para problemas de comportamento.

8. DIAS, CUNHA, Jogo de perguntas de busca com figuras diversas (PBFD) ( ENUMO, $2011 \quad$ Gera e Linhares ,1998) Jogo de perguntas de busca com figuras diversas (PBFD) (Gera e Linhares ,1998) jogo de perguntas de busca com figuras geométricas para crianças com deficiência visual (PBFG-DJV) (Enumo \& Batista, 2000)/ Teste de Desempenho Escolar (TDE) (Stein, 1994) Escala Colúmbia de maturidade intelectual (Burgmeister, Blum\&Lorge, 1967) / a mediação oferecida na fase de assistência está diretamente relacionada à melhora no desempenho de alunos mais comprometidos funcionalmente

9. PAULA, ENUMO,2007 Children's Analogical Thinking Modifiability Test - CATM/ Escala de Comunicação Pré-Verbal - ECPV (Kiernan \& Reid, 1988, apud Paula, 2004) Lista de Avaliação do Vocabulário Expressivo - LAVE (Rescorla, 1989, traduzida por Capovilla; Capovilla, 1997), Escala Columbia de Maturidade Intelectual (Rodrigues \& Rocha, 1999) Teste de Vocabulário por Imagens Peabody TVIP (Capovilla \& Capovilla, 1997), (Capovilla, Duduchi \& Macedo, 1997a), Matrizes Progressivas Coloridas de Raven Escala Especial (Raven, 1974, apud Angelini et al., 1999)/ A avaliação assistida foi reconhecida uma relevante ferramenta de diagnóstico psicológico, complementar à abordagem psicométrica tradicional, para crianças com déficits comunicativos.

10. GERA, LINHARES, Jogo de Perguntas Busca com Figuras Diversas-PBFD (Gera, 2004 2001);/ Teste de Raven Escala Especial (Angelini, Alves, Custódio \& Duarte, 1987).

Embora as pesquisas revisadas se utilizem de delineamentos de pesquisa experimental intrasujeitos, por vezes, o número de participantes é maior que os empregados nesse tipo de investigação. Dentre os principais resultados encontrados nessa pesquisa de revisão, destacam-se a melhora no desempenho da criança com PC, após o processo de mediação. Essa melhoria ocorreu 
em função do tipo e intensidade da mediação oferecida, da natureza das barreiras cognitivas e da distância psicológica entre o conteúdo do ensino e o conteúdo de desempenho nos testes.

Nos artigos analisados pode-se observar que há melhora no desempenho da fase inicial. A aprendizagem adquirida nesta fase é transferida para as fases subsequentes, sugerindo que apesar da complexidade das tarefas nas mudanças de fases a generalização da aprendizagem ocorre com mais facilidade. Provavelmente, fatores como a possibilidade de contar com modelos já conhecidos de outras fases e a utilização dos mesmos arranjos das fases anteriores pode ter contribuído para este resultado (GERA; LINHARES, 2004; DIAS; CUNHA; ENUMO, 2011).

Há ainda a transferência das estratégias mediadas para novos contextos de resolução de problemas. Isto gera uma medida do potencial de aprendizagem que, de outro modo, seria difícil obter em uma testagem normativa, como ocorre, por exemplo, na avaliação de examinandos que resistem à avaliação. Esses, evidenciam graves problemas de comunicação e são afetados por barreiras psicológicas (por exemplo, o autoconceito negativo como aprendiz), para a expressão das habilidades requeridas em tarefas cognitivas. Trata-se de procedimento que fornece previsões mais otimistas do que aquelas produzidas por meio dos testes estáticos (HAYWOOD; TZURIEL, 2002; TZURIEL, 2001).

As autoras Dias, Cunha e Enumo (2011), em sua pesquisa, apresentaram a avaliação assistida ou dinâmica como uma nova perspectiva baseada na abordagem sociointeracionista. Para essas autoras a avaliação assistida ou dinâmica trouxe avanços para a área de avaliação cognitiva, oferecendo indicadores do potencial de aprendizagem. A pesquisa realizada por estas autoras verificou a adequação de provas cognitivas assistidas, que incluem uma fase intermediária de assistência em crianças com dificuldade de aprendizagem (DA) e deficiência visual (DV). Para tanto, utilizou-se do jogo de perguntas de busca com figuras diversas (PBFD) e o jogo de perguntas de busca com figuras geométricas para crianças com deficiência visual (PBFG-DV). Esses jogos investigam as estratégias utilizadas ao se elaborarem questões de busca de informação com restrição de alternativa, em situação de resolução de problemas. Ao final do jogo, obtém-se um perfil de desempenho cognitivo. As provas assistidas foram sensíveis em identificar a variação de desempenho das crianças, mostrando-se adequadas para a avaliação de habilidades cognitivas de crianças com diferentes necessidades educativas especiais.

Gera e Linhares (2004) realizaram um estudo cujo objetivo foi avaliar indicadores de potencial cognitivo de crianças com queixa de dificuldades de aprendizagem, utilizando procedimento combinado de avaliação psicométrica (Raven) e avaliação assistida (Jogo de Perguntas de Busca com Figuras Diversas - PBFD). Foram avaliadas 34 crianças (8 - 11 anos). O PBFD é delineado em fases, inicial sem ajuda (SAJ), assistência (ASS), manutenção imediata (MAN), manutenção posterior (MANP), transferência I (TRF-I) e transferência II (TRF-II) e avalia desempenho, operações cognitivas e comportamento. Da amostra, 59\% das crianças classificaram-se abaixo da média no Raven. Comparando-se ASS, MAN, MANP, TRF-I e TRF-II em relação a SAJ, verificou-se aumento significativo das perguntas relevantes e do número de acertos (exceção TRF-I). Houve aumento significativo do número de crianças com operações cognitivas positivas em ASS, MAN, TRF-I e TRF- 
II comparado a SAJ. Os comportamentos positivos ocorreram em pelo menos $62 \%$ em todas as fases.

Quanto à manutenção e transferência da aprendizagem, os estudos apresentaram heterogeneidade nos resultados, uma vez que algumas crianças dos estudos conseguiram manter e transferir o repertório aprendido enquanto outras não (Gera \& Linhares (2004). Esses estudos vão de encontro com os achados de pesquisa de Brown e Ferrara (1985) e por Ferrara, Brown e Campione (1986), nos quais verificaram-se variações intra-grupo quanto ao desempenho na transferência da aprendizagem.

Os estudos analisados confirmam o que Lidz (1992) preconiza no que se refere a mediação oferecida na fase de assistência. Esta está diretamente relacionada à melhora no desempenho de alunos mais comprometidos funcionalmente. Autores como Klein (1996), Lidz (1992) e Tzuriel (2001) também confirmam os efeitos positivos da mediação na mudança de desempenho durante e após a avaliação.

O uso de estratégias de intervenção na população de crianças com deficiência deve considerar que as disfunções cognitivas nos processos de aprendizagem podem ser modificadas estruturalmente, mediante o envolvimento do mediador na elaboração de contextos motivadores ao aprendiz. Além disso, que proponham à criança o desafio de se engajar em novas e diferentes situações de aprendizagem. Ainda que haja limitações impostas pela deficiência, a possibilidade de mudança cognitiva estará sempre presente.

Os estudos descritos corroboram outras investigações da área (HAYWOOD, 2008; HAYWOOD; LIDZ, 2007; HAYWOOD; TZURIEL, 2002; TURRINI, 2011; TZURIEL, 2001), mostrando que os procedimentos e instrumentos assistidos contribuem para uma avaliação completa dos diversos programas de intervenção educacionais e cognitivos, especialmente para crianças com deficiência.

Vale ressaltar mais uma vez que as avaliações são importantes quando contribuem para o desenvolvimento de estratégias adequadas que melhorem as condições psicológicas, educacionais, afetivas e sociais de nossas crianças.

\section{Conclusão}

As pesquisas sobre a Avaliação Assistida, nos últimos dez anos, vêm delineando um campo bastante promissor devido às suas características inovadoras que a distinguem de uma avaliação tradicional, beneficiando assim a população com algum tipo de deficiência, como neste estudo, isto é, aquelas com Paralisia Cerebral.

Neste tipo de avaliação, as variações intragrupo na população de crianças com deficiência são consideradas, permitindo assim o emprego de intervenções individualizadas. Apesar das limitações apontadas, mesmo os críticos da área a descrevem como uma wonderful idea (Sternberg \& Grigorenko, 2002), dada a possibilidade de fornecer informações mais úteis e válidas para pais, 
educadores e psicólogos, particularmente nos campos da Educação Especial e Psicologia do Desenvolvimento.

No entanto, na realidade brasileira, os resultados positivos ainda conferem à Avaliação Assistida um status modesto, considerando-se o fato de ser uma área de conhecimento emergente, com aplicação mais restrita a poucos grupos de pesquisa. Todavia, é uma modalidade coerente com a direção dos novos rumos de uma avaliação que gera um amplo volume de informações sobre os processos de resolução de desafios cognitivos. Somam-se a isso, as estratégias adequadas à aprendizagem na medida em que fornece subsídios teóricos e metodológicos para a implantação de diferentes programas de intervenção, para crianças com Deficiência. Nesta proposta, será possível fornecer um conjunto de estratégias mais eficazes para ampliar ferramentas cognitivas, criativas e de comunicação que garanta uma real inclusão escolar e social de crianças com deficiência.

Os indicadores proporcionados pela Avaliação Assistida, apresentados neste estudo poderão subsidiar a elaboração de estratégias adequadas para que essas crianças adquiram motivação para aprender. Assim, ao final dessas apreciações, alcançamos a proposta principal de divulgar a relevância da temática através de uma síntese do conhecimento produzido nos últimos 10 anos sobre as melhores práticas em Avaliação Cognitiva Assistida e no atendimento de crianças diagnosticadas com Paralisia Cerebral - PC. Possivelmente, este estudo suscitará a curiosidade e o interesse de outros pesquisadores, para que possam, talvez, incrementar a área com futuras investigações.

Em outras palavras, aguardamos novos tempos no campo da Avaliação Assistida e seu impacto direto nas medidas educacionais para uma sociedade mais inclusiva. A integração de áreas tão promissoras, como esta e a Comunicação Alternativa poderão auxiliar no desenvolvimento de intervenções psicológicas mais sensíveis e eficazes, contribuindo para melhorar a Qualidade de Vida das crianças com PC e outras deficiências.

\section{Referências}

AWAAD, Y. et al. Functional assessment following intrathecal baclofen therapy in children with spastic cerebral palsy. J Child Neurol, v.18, n. 1, p. 26-34, 2003.

BROWN, A. L.; FERRARA, R. A. Diagnosing zones of proximal development. In: J.V. Wertsch (Ed.). Culture communication and cognition: Vygotskian perspectives. Cambridge: Cambridge University Press, 1985.

BLUME, H.K.; LOCH, C.M. Li IC. Neonatal Encephalopathy and Socioeconomic Status. Arch Pediatr Adolesc Med, v.161, n.7, p. 663-668, 2007.

CAMPIONE, J.C. Assisted assessment: A taxonomy of approaches and an outline of strengths and weaknesses. Journal of Learning Disabilities, 22, 151-165, 1989.

CANS, C. Surveillance of cerebral palsy in: Europe: a collaboration of cerebral palsy surveys and registers. Dev Med Child Neurol, v. 42, n. 816, 2000.

CENCI, A.; COSTAS, F. A. T. Mediação e conceitos cotidianos: os aportes de Feuerstein e Vygotsky para investigar as dificuldades de aprendizagem. Psicologia em Revista, v. 19, n. 2, p. 250-270, 
2013. Disponível em: < https://dx.doi.org/DOI - 10.5752/P.1678-9563.2013v19n2p250> Acesso em: 10 out. 2017.

CORREIA, L. M.; TONINI, A. Avaliar para intervir: um modelo educacional para alunos com necessidades especiais. Revista Educação Especial, Marília, v.25, n.44, p. 367-382, 2012. Disponível em: <http://cascavel.ufsm.br/revistas/ojs2.2.2/index.php/educacaoespecial/issue/view/434/showToc>. Acesso em: 06 set. 2015.

CURTO, L. M.; MORILLO, M. M.; FEIXIDÓ, M. M. Escrever e ler: como as crianças aprendem e como o professor pode ensiná-las a escrever e ler. Tradução: Ernani Rosa. Porto Alegre: Artmed, 2000.

DZIENKOWSKI, R.C. et al. Cerebral palsy: a comprehensive review. Nurse Pract, v. 2, n. 1, p. 4559, 1996.

ENUMO, S. R. F. Avaliação assistida para crianças com necessidades educativas especiais: um recurso auxiliar na inclusão escolar. Revista Brasileira de Educação Especial, n. 11, p. 335-354, 2005.

ENUMO, S. R. F.; BATISTA, C. G. Avaliação cognitiva assistida com o Jogo de Perguntas de Busca com Figuras Geométricas para Crianças com Deficiência Visual (PBFG-DV). In: LINHARES, M. B. M.; ESCOLANO, A. C. M.; ENUMO, S. R. F. (Orgs.). Avaliação cognitiva assistida: fundamentos, procedimentos e aplicabilidade . São Paulo: Casa do Psicólogo, 2006. p. 137-159.

ENUMO, S. R. F.; BATISTA, C. G. Avaliação assistida de habilidades cognitivas de crianças com deficiência visual por Jogo de Perguntas de Busca com Figuras Geométricas para Crianças com Deficiência Visual (PBFG-DV). In: LINHARES, M. B. M.; ESCOLANO, A. C. M.; ENUMO, S. R. F. (Orgs.). Avaliação assistida: Fundamentos, procedimentos e aplicabilidade São Paulo: Casa do Psicólogo, 2006. p. 87-101.

FONSECA, V. D A. Aprender a Aprender: a educabilidade cognitiva. Porto Alegre: Artmed, 1998. FEUERSTEIN, R.; FEUERSTEIN, S. Mediated learning experience: A theorical review. In: Feuerstein, R.; Klein, P. S.; Tannenbaum, A. J. (Eds.). Mediated Learning Experience (MLE): Theorical, psychological and learning implications. London: International Center for Enhancement of Learning Potential (ICELP), 1991. p. 3-51.

JOHNSON, A. Prevalence and Chacacteristics of Children With Cerebral Palsy in Europe. Dev Med Child Neurol, n. 44, p. 633-640, 2002.

KAVCIC, A.; VODUSEK, D.B. A historical perspective on cerebral palsy as a concept and a diagnosis. Eur J Neurol., v.12, n. 8, p. 582-7, 2005.

KNOX, V.; EVANS, A. L. Evaluation of the functional effects of a course of Bobath therapy in children with cerebral palsy: a preliminary study. Dev Med Child Neurol., v. 44, n. 7, p. 447-60, 2002.

LAYTON, C. A.; LOCK, R. H. Determining learning disabilities in students with low vision. Journal of Visual Impairment \& Blindness, May, 288-298., 2001.

LINHARES, M.B.M. Avaliação assistida em crianças com queixa de dificuldades de aprendizagem. Temas de Psicologia, n. 1, p. 17-32, 1996.

MANOEL, E.J.; OLIVEIRA, J.A. Motor developmental status and task constraint in overarm throwing. Journal of Human Movement Studies, n. 39, p. 359-78, 2000.

MORENO-DE-LUCA, A. D.H.; LEDBETTER, C.L.; MARTIN. Genetic insights into the causes and classification of the cerebral palsies. Lancet Neurol., n. 11, p. 283-292, 2012.

MURPHY, D.J.; HOPE, P.L.; JOHNSON, A. Neonatal risk factors for cerebral palsy in very preterm babies: case- control study. BMJ, n. 314, p. 404-408, 1997. 
NORONHA, A.P.P. et al. Cartilha Avaliação Psicológica. [S.I.]: CFP, 2013.

PALISANO, R. et al. Development and reliability of a system to classify gross motor function in children with cerebral palsy. Dev Med Child Neurol.;39(4):214-23, 1997.

PAULA, K.M.P.; ENUMO, S.R.F. Avaliação assistida e comunicação alternativa: procedimentos para a educação inclusiva. Revista Brasileira de Educação Especial, Marília, v. 13, n. 1, p. 3- 26, 2007.

PAURA, A.C. Estudo de vocábulos para proposta de instrumento de avaliação do vocabulário de crianças não-oralizadas. 2009. 135 f. Tese (Doutorado em Educação) - Faculdade de Filosofia e Ciências, Universidade Estadual Paulista, Marília, 2009.

ROBERTSON, C.M.T.; WATT, M.; YASUI, Y. Changes in the Prevalence of Cerebral Palsy for Children Born Very Prematurely Within a Population-Based Program Over 30 Years. JAMA, v.297, n. 24, p. 2733-2740, 2007.

ROSENBAUM, P. et al. Report: the definition and classification of cerebral palsy April Dev Med Child Neurol., v. 49, n. 9, 2006.

SANTA MARIA, M. R.; LINHARES, M. B. M. Avaliação cognitiva assistida de crianças com indicações de dificuldades de aprendizagem escolar e deficiência mental leve.In: reflexão e crítica, v. 12, p. 395-417, 1999.

Psicologia:

SAWAIA, B. (Org.) As artimanhas da inclusão: análise psicossocial e ética da desigualdade social. Petrópolis: Vozes, 2001.

SCHWARTZMAN, J.S. Paralisia cerebral. Arquivos Brasileiros de Paralisia Cerebral, v. 1, n. 1, p. 4-17, 2004.

SERDAROGLU, A. et al. Prevalence of cerebral palsy in Turkish children between the ages of 2 and 16 years. Dev Med Child Neurol, n. 48, p. 413-416, 2006.

SILVEIRA, R.C.; PROCIANOY, R.S. Lesões Isquêmicas Cerebrais no Recém-Nascido Pré-Termo de Muito Baixo Peso. J Pediatr, Rio de Janeiro, v. 81, n. 1 Supl, p. 23-32, 2005.

STANLEY, F.J.; WATSON, L. Trends in perinatal mortality and cerebral palsy in Western Australia, 1967 to 1985. BMJ, n.304, p.1658-1663, 1992.

SWANSON, H.L. Effects of dynamic testing on the classification of learning disabilities: the predictive and discriminant validity of the Swanson - cognitive processing test (S-CPT). Journal of Psychoeducational Assessment, v. 13, p. 204-229, 1995.

TZURIEL, D. Dynamic assessment of young children. New York: Kluwer Academic/Plenum Publishers, 2001.

TZURIEL, D.; KLEIN, P. S. Analogical thinking modifiability in disadvantaged, regular, special education, and mentally retarded children. Journal of Abnormal Child Psychology, 13, 539-552, 1985.

TZURIEL, D.; KLEIN, P. S. The Children's Analogical Thinking Modifiability Test: Instruction manual. Ramat-Gan, Israel: School of Education Barllan University, 1990.

VOHR, B. R. et al. Spectrum of Gross Motor Function in Extremely Low Birth Weight Children With Cerebral Palsy at 18 Months of Age. Pediatrics, v. 116, p. 123-129, 2005.

VYGOTSKY, L. S. A Formação Social da Mente. São Paulo: Martins Fontes, 1984.

VYGOTSKY, L. S. Pensamento e linguagem. São Paulo: Martins Fontes, 1993. 
WESTBOM, L. ; HAGGLUND, G.; NORDMARK, E. Cerebral palsy in a total population of 4-11 year olds in southern Sweden. Prevalence and distribution according to different CP classification systems. BMC Pediatrics, v. 7, n. 41, p. 1-8, 2007.

WORLD HEALTH ORGANIZATION. International classification of functional and disability, beta2 version. Geneva: WHO, 1999.

ZANINI, G.N.F.; CEMIN, S.N.; PERALLES. Paralisia cerebral: causas e prevalências. Fisioter Mov., v. 3, p. 375-381, 2009. 